\title{
Einfluss systematischer Umwelteffekte auf die Milchleistungsmerkmale bei Bunten Deutschen Edelziegen
}

\begin{abstract}
Title of the paper: Influence of systematic environmental effects on milk performance traits in German Improved Fawn

The objectives of this study were to analyse the influence of fixed effects on milk traits of German Improved Fawn. The analysis was based on 27,778 test day records of 1,848 German Improved Fawn with 3,574 lactation records. The milk records were sampled between 1988 and 2002 from 229 flocks in Lower Saxony, Saxony and Baden-Wuerttemberg. The average daily milk yield was $2.87 \pm 1.20 \mathrm{~kg}$ with a fat content of $3.08 \pm 0.54 \%$ and a protein content of $3.38 \pm 0.95 \%$. Somatic cell count (SCC) was transformed into somatic cell score (SCS). Mean SCS was $5.49 \pm 1.93$. The average lactation length was $234.1 \pm 76.4$ days. The analysis of variance showed a significant influence of lactation number, stage of lactation, year of lambing and month of lambing on all analysed milk production traits. Milk yield was highest in the fourth lactation number and fat as well as protein content in the 7th to 13th lactation number. Litter size had a significant influence on milk and fat yield and on fat and protein content. The region significantly influenced protein yield and fat and protein content of German Improved Fawn but had no effect on milk and protein yield and SCS. Animals from Baden-Wuerttemberg reached the highest milk protein content but the lowest milk fat content.
\end{abstract}

Key Words: goats, milk performance traits, systematic effects

\section{Zusammenfassung}

Ziel der vorliegenden Arbeit war es, den Einfluss systematischer Umwelteinflüsse auf die Milchleistungsmerkmale Bunter Deutscher Edelziegen zu untersuchen. Das untersuchte Datenmaterial bestand aus 27.778 Testtagsergebnissen von 1.848 milchleistungsgeprüften Bunten Deutschen Edelziegen mit 3.574 Laktationsleistungen aus den Jahren 1988-2002. Die Tiere wurden in 229 Betrieben im Bereich des Ziegenzuchtverbandes Niedersachsen, des Sächsischen Schaf- und Ziegenzuchtverbandes und des Ziegenzuchtverbandes Baden-Württemberg gehalten. Die durchschnittliche Milchleistung betrug 2,87 \pm 1,20 kg Milch pro Tag mit einem Fettgehalt von 3,08 \pm 0,54\% und einem Eiweißgehalt von 3,38 $\pm 0,95 \%$. Die somatische Zellzahl (SCC) wurde für die Auswertung zum somatischen Zellgehalt (SCS) transformiert und lag durchschnittlich bei 5,49 \pm 1 ,93. Die Laktationsdauer betrug im Mittel 234,1 \pm 76,4 Tage. Die Varianzanalyse zeigte einen signifikanten Einfluss der Laktationsnummer, des Laktationsstadiums, des Ablammjahres und des Ablammmonats auf alle untersuchten Milchleistungsmerkmale. Die höchste Milchmenge erreichten die Ziegen in der vierten Laktation, die höchsten Fettund Eiweißgehalte in der 7. - 13. Laktation. Tiere mit Ablammungen im Januar und Februar wiesen eine höhere Milchmenge und einen höheren SCS, aber geringere Fett- und Eiweißgehalte auf. Die Anzahl lebend geborener Lämmer erwies sich als signifikant für die Milch- und Fettmenge sowie den Fett- und Eiweißgehalt, nicht jedoch auf die Eiweißmenge und den SCS der Bunten Deutschen Edelziegen. Ziegen mit mehr als 2 Lämmern hatten eine höhere Milchmenge, aber einen geringeren Fett- und Eiweißgehalt als Tiere mit 1 oder 2 Lämmern. Die Region hatte einen signifikanten Effekt auf die Eiweißmenge und den Fett- sowie Eiweißgehalt. Dagegen blieben die Milch- und Fettmenge sowie der SCS von der Region unbeeinflusst. Die Tiere aus Baden-Württemberg wiesen den höchsten Eiweiß-, aber niedrigsten Fettgehalt in der Milch auf.

Schlüsselwörter: Milchziegen, Milchleistungsmerkmale, systematische Effekte

1. Einleitung

Nach 1945 nahm mit steigendem Wohlstand die Zahl in Deutschland gehaltener Ziegen kontinuierlich ab und erreichte 1977 mit 36.000 Tieren ihren Tiefststand. In den 
letzten Jahren stieg hingegen das Interesse an der Ziegenhaltung und die Nachfrage nach Ziegenmilch und Ziegenmilchprodukten deutlich an, so dass inzwischen ca. 160.000 Ziegen in Deutschland gehalten werden. Von diesen stellen die Milchziegenrassen Bunte und Weiße Deutsche Edelziegen den größten Anteil dar. Immer häufiger werden in spezialisierten Betrieben größere Ziegenherden zur Milchproduktion mit Abgabe der Milch an Molkereien oder Direktvermarktung von Milchprodukten gehalten.

Die Bunte Deutsche Edelziege ist die in Deutschland am weitesten verbreitete Milchziegenrasse. Ihre durchschnittliche Milchleistung beträgt $750 \mathrm{~kg}$ in 280-300 Melktagen mit 3,4\% Fett und 3\% Eiweiß. Es können Spitzenleistungen von $1900 \mathrm{~kg}$ erbracht werden. Die Fruchtbarkeit beträgt 1,8 bis 2,0 lebend geborene Lämmer je Ziege und Jahr. Bei entsprechender Haltung und Fütterung können die Tiere bis zum Alter von 12 bis 15 Jahren gute Leistungen erbringen (GALL, 2001; SAMBRAUS, 2001).

Trotz der zunehmenden Bedeutung der Bunten Deutschen Edelziegen für die Milchproduktion liegt bisher lediglich eine Arbeit zur Schätzung genetischer Parameter (BÖMKES et al., 2004), jedoch keine Analyse für systematische Effekte auf die Milchleistungsmerkmale für diese Milchziegenrasse vor.

Ziel dieser Arbeit ist es deshalb, die Auswirkung verschiedener Umwelteinflüsse auf die Milchmenge und die Milchinhaltstoffe der Bunten Deutschen Edelziegen zu untersuchen.

\section{$2 . \quad$ Material und Methode}

Die Daten wurden vom VIT Verden, dem Landeskontrollverband Sachsen und der Rinderunion Baden-Württemberg zur Verfügung gestellt. Es handelte sich um nach den IKLT-Richtlinien gewonnene Probemelkergebnisse, die sich aus der Milchmenge des Testtages, dem Fett- und Eiweißgehalt und teilweise der somatischen Zellzahl zusammensetzten. Aus Baden-Württemberg lagen keine Angaben zur somatischen Zellzahl vor.

Der Gehalt an somatischen Zellen pro ml Ziegenmilch (SCC) wurde für die Auswertungen zum Somatic Cell Score (SCS) transformiert. Dieser berechnete sich nach folgender Formel:

$$
\mathrm{SCS}=\log _{2}\left(\mathrm{SCC} / 10^{5}\right)+3
$$

In der Tabelle 1 sind die Anzahl der Betriebe mit Milchleistungsprüfung sowie die Anzahl geprüfter Tiere, Laktationen und Testtagsergebnisse dargestellt.

Tabelle 1

Anzahl der geprüften Tiere, Laktationen, der Testtagsergebnisse (PM) und Betriebe nach Verbänden (Number of animals recorded, lactation and test day results and herds by goat breeding organisations)

\begin{tabular}{ccccc}
\hline Verband & Anzahl Tiere & $\begin{array}{c}\text { Anzahl } \\
\text { Laktationen }\end{array}$ & $\begin{array}{c}\text { Anzahl } \\
\text { PM }\end{array}$ & Anzahl Betriebe \\
\hline Niedersachsen & 195 & 376 & 2.857 & 11 \\
Sachsen & 85 & 111 & 826 & 12 \\
Baden-Württemberg & 1.568 & 3.087 & 24.095 & 206 \\
\hline Gesamt & 1.848 & 3.574 & 27.778 & 229 \\
\hline
\end{tabular}

Die Probemelkergebnisse aus Baden-Württemberg reichten von 1988 bis 2001. Aus Niedersachsen und Sachsen wurden Ergebnisse aus den Jahren 1999 bzw. 2000 bis 2002 untersucht. Insgesamt wurden 3.574 Laktationen von 1.848 Bunten Deutschen 
Edelziegen berücksichtigt. Während von 588 Tieren nur jeweils eine Laktationsleistung vorlag, wurden 1.260 Tiere mehrmals geprüft. Insgesamt standen aus den untersuchten Laktationen 27.778 Probemelkergebnisse zur Verfügung. Die milchleistungsgeprüften Bunten Deutschen Edelziegen wurden in 229 Betrieben gehalten, die sich zu 90\% im Bereich des Ziegenzuchtverbandes Baden-Württemberg befanden. 4,8\% der Betriebe lagen im Bereich des Ziegenzuchtverbandes Niedersachsen, und 5,2\% der Betriebe gehörten zum Sächsischen Schaf- und Ziegenzuchtverband. Die Bunten Deutschen Edelziegen brachten zwischen ein und vier Lämmer zu Welt. Am häufigsten traten Zwillingsgeburten auf (58,8\%), gefolgt von Einlingen (25,1\%) und Drillingen (15,1\%). Vierlingsgeburten waren sehr selten (1,0\%). Die Milchziegen befanden sich in der ersten bis dreizehnten Laktation. Während mit 23,4\% die meisten Milchleistungen von Ziegen in der ersten Laktation vorlagen, nahm die Häufigkeit in den folgenden Laktationen kontinuierlich ab. Dies ist auf ein Ausscheiden von Tieren aus den Betrieben auf Grund von Krankheit oder zu geringer Leistung zurückzuführen. Die zehnte bis dreizehnte Laktation erreichten 2,6\% der Bunten Deutschen Edelziegen. Da Ziegen ein saisonales Fortpflanzungsverhalten zeigen, lammten sie überwiegend in den Winter- und Frühlingsmonaten ab. So fielen 77,7\% der Lammungen in die Monate Januar bis März.

Die Bedeutung der erfassten systematischen Einflussfaktoren auf die Milchleistung wurde mittels der Prozedur MIXED von SAS, Version 8.2 (SAS Institute, Cary, N.C., USA, 2002) untersucht. Dazu wurde eine multiple Varianzanalyse mit dem folgenden gemischten Modell unter Verwendung von Residual Maximum Likelihood (REML) durchgeführt.

Modell für die Milchleistungsmerkmale der Bunten Deutschen Edelziege

$$
\begin{aligned}
& \mathrm{Y}_{\mathrm{ijklmnopqr}}=\mu+\mathrm{R}_{\mathrm{i}}+\mathrm{S}_{\mathrm{j}}+\mathrm{J}_{\mathrm{k}}+\mathrm{Z}_{\mathrm{l}}+\mathrm{L}_{\mathrm{m}}+\mathrm{PI}_{\mathrm{n}}+\mathrm{h}(\mathrm{R})_{\mathrm{io}}+\mathrm{t}_{\mathrm{p}}+\mathrm{b}_{1} * \mathrm{DIM}_{\mathrm{q}}+\mathrm{b}_{2} *\left(\mathrm{DIM}_{\mathrm{q}}\right)^{2}+ \\
& \mathrm{b}_{3} * \log \operatorname{DIM}_{\mathrm{q}}+\mathrm{b}_{4} *\left(\log \operatorname{DIM}_{\mathrm{q}}\right)^{2}+\mathrm{e}_{\mathrm{ijklmnopqr}}
\end{aligned}
$$
Eiweißgehalt und den SCS des ijklmnopqr-ten Tieres

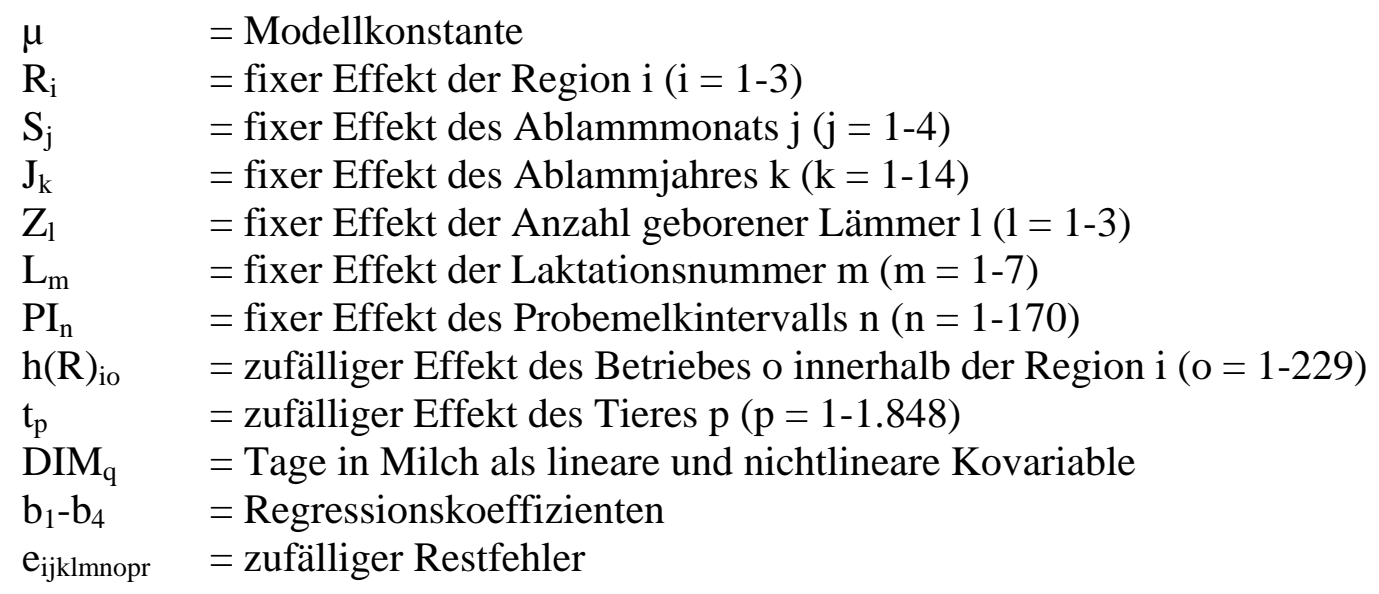

Die milchleistungsgeprüften Tiere wurden in Betrieben im Gebiet des Niedersächsischen Landesziegenzuchtverbandes, des Sächsischen Schaf- und Ziegenzuchtverbandes und des Ziegenzuchtverbandes Baden-Württemberg gehalten. Der Standort des Tieres ging als fixer Effekt der Region in die Auswertung ein. Auf Grund der unterschiedlichen Verteilung der Lammungen innerhalb des Jahres wurden die Ablamm- 
monate zu Klassen zusammengefasst. Dabei bildeten die Monate Januar, Februar und März ebenso wie die Monate April bis Dezember jeweils eine Klasse. Während die meisten Tiere im Januar ablammten $(n=825)$, brachten in den Monaten April bis Dezember insgesamt 701 Bunte Deutsche Edelziegen ihre Lämmer zur Welt. Die Anzahl der geborenen Lämmer variierte zwischen ein und vier Lämmern. Am häufigsten waren Zwillingsgeburten $(n=1844)$. Drillinge und Vierlinge wurden wegen ihres selteneren Auftretens zu einer Klasse zusammengefasst $(n=494)$. Die milchleistungsgeprüften Tiere befanden sich in der ersten bis dreizehnten Laktation. Am häufigsten wurden Bunte Deutsche Edelziegen in der ersten Laktation beprobt $(n=688)$. Die 458 Tiere in der siebten bis dreizehnten Laktation wurden zu einer Klasse zusammengefasst. Am geringsten war die Klasse mit Tieren in der sechsten Laktation besetzt ( $\mathrm{n}=$ 234). Da zu wenig Bunte Deutsche Edelziegen am gleichen Tag geprüft worden waren, konnte der Testtag nicht direkt als fixer Faktor in das Modell einfließen, sondern wurde zu 30-tägigen Probemelkintervallen zusammengefasst. Auf Grund der geringen Anzahl an Milchleistungsprüfungen in den Wintermonaten, wurden bis zu 4 dieser Intervalle zu einem Intervall zusammengefasst. So ergaben sich 170 Probemelkintervalle. Da ein Betrieb immer genau einem Landesziegenzuchtverband zuzuordnen war, wurde der Effekt des Betriebes innerhalb der Region genestet und ging als zufälliger Effekt in das Modell ein. Die Tage in Milch stellten die Differenz zwischen dem Ablammdatum und dem Probemelkdatum dar und wurden als lineare, quadratische, logarithmische und logarithmisch-quadrierte Kovariable im Modell berücksichtigt.

\section{Ergebnisse}

Im Durchschnitt dauerte die Laktation der Bunten Deutschen Edelziegen 234,1 \pm 76,4 Tage. Während dieser Zeit produzierten die Tiere durchschnittlich 2,87 $\pm 1,20 \mathrm{~kg}$ Milch pro Tag mit einem Fettgehalt von 3,08 $\pm 0,54 \%$ und einem Eiweißgehalt von 3,38 \pm 0,95\%. Der SCS betrug im Durchschnitt 5,49 $\pm 1,93$.

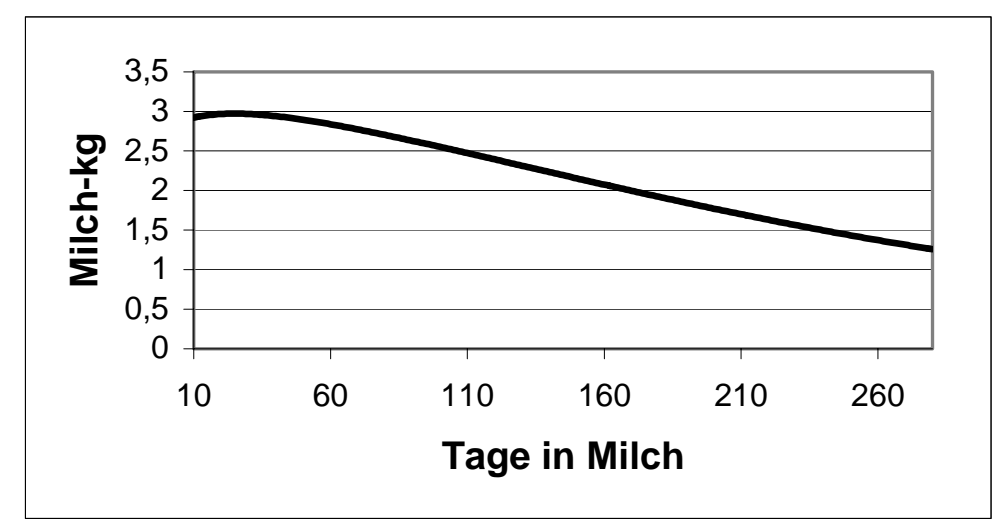

Abb. 1: Laktationskurvenverlauf der Milchmenge (kg) der Bunten Deutschen Edelziegen (Lactation curve for milk yield (kg) of German Improved Fawn)

Die Laktationskurve für die Milchmenge fiel nach einem sehr geringen Anstieg zu Beginn der Laktation kontinuierlich ab (Abb. 1). Umgekehrt verhielten sich der Fettgehalt und der Eiweißgehalt, die nach einem Abfall am Anfang der Laktation bis zum Ende der Laktation stetig anstiegen (Abb. 2 und 3). Die Laktationskurve des SCS stieg zunächst leicht an, blieb dann zunächst auf dem erreichten Niveau und fiel in der zweiten Hälfte der Laktation wieder geringfügig ab (Abb. 4). 


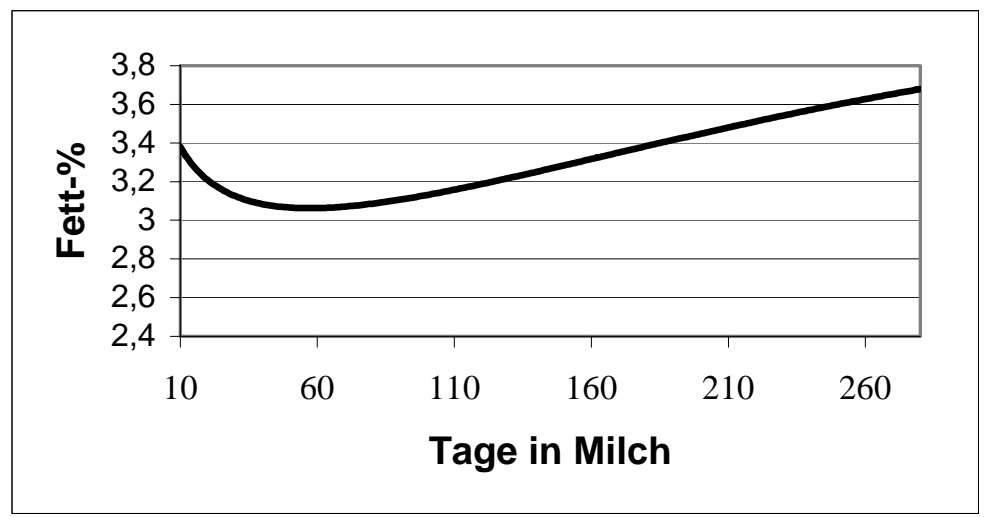

Abb. 2: Laktationskurvenverlauf für den Fettgehalt der Bunten Deutschen Edelziegen (Lactation curve for fat content of German Improved Fawn)

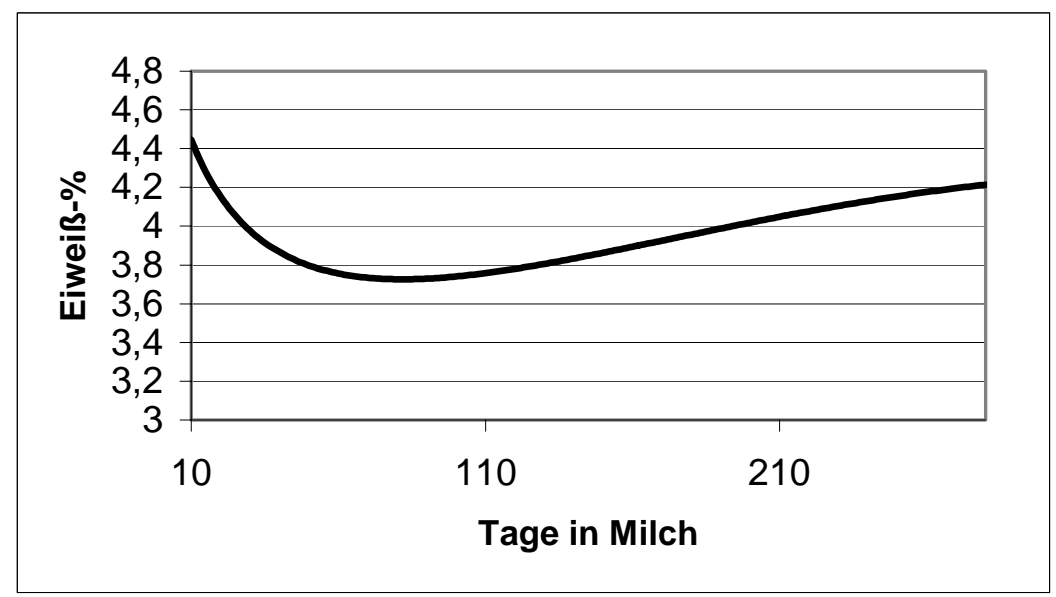

Abb. 3: Laktationskurvenverlauf für den Eiweißgehalt der Bunten Deutschen Edelziegen (Lactation curve for protein content of German Improved Fawn)

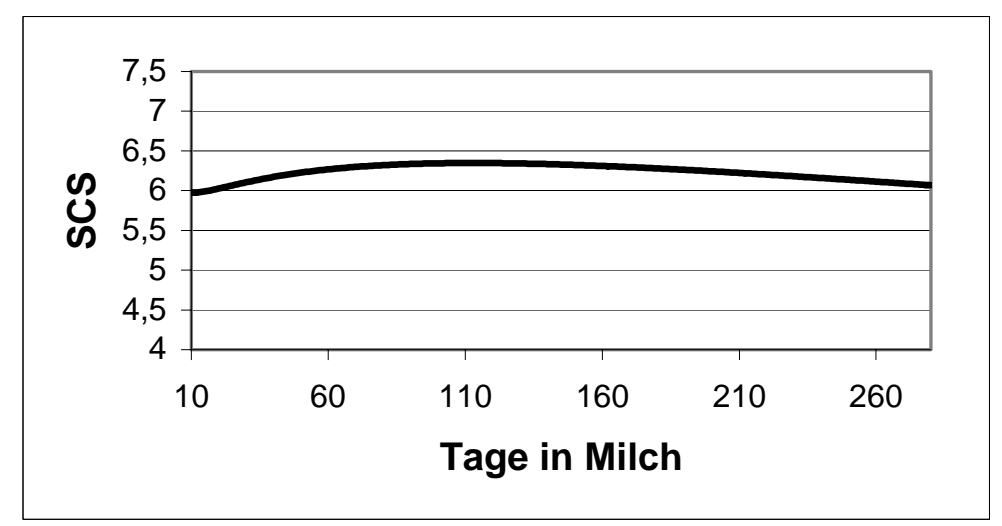

Abb. 4: Laktationskurvenverlauf für den somatischen Zellgehalt (SCS) der Bunten Deutschen Edelziegen (Lactation curve for somatic cell score (SCS) of German Improved Fawn)

Die Laktationsnummer, der Ablammmonat, das Ablammjahr und das Probemelkintervall hatten einen signifikanten Einfluss auf alle untersuchten Merkmale (Tab. 2). Weiterhin hatte die Region einen signifikanten Effekt auf die Eiweißmenge sowie den Fett- und Eiweißgehalt der Bunten Deutschen Edelziegen. Die Anzahl lebend geborener Lämmer übte außerdem einen signifikanten Einfluss auf die Milch- und Fettmenge 


\section{sowie den Fett- und Eiweißgehalt aus. Der SCS wurde weder von der Region noch von der Anzahl lebend geborener Lämmer signifikant beeinflusst.}

Tabelle 2

Signifikanz der systematischen Einflussfaktoren auf die Milchleistungsmerkmale der Bunten Deutschen Edelziegen (Significant influences of fixed effects on milk production traits of German Improved Fawn)

\begin{tabular}{ccccccc}
\hline \multirow{2}{*}{ Merkmal } & \multicolumn{5}{c}{ Einflussfaktoren } \\
\cline { 2 - 7 } & Region & $\begin{array}{c}\text { Anzahl } \\
\text { Lämmer }\end{array}$ & $\begin{array}{c}\text { Ablamm- } \\
\text { monat }\end{array}$ & $\begin{array}{c}\text { Ablamm- } \\
\text { Jahr }\end{array}$ & $\begin{array}{c}\text { Laktations- } \\
\text { nummer }\end{array}$ & $\begin{array}{c}\text { Probemelk- } \\
\text { intervall }\end{array}$ \\
\hline Mkg & n.s. & $* * *$ & $* * *$ & $* * *$ & $* * *$ & $* * *$ \\
Ekg & $*$ & n.s. & $* * *$ & $* * *$ & $* * *$ & $* * *$ \\
Fkg & n.s. & $* * *$ & $* * *$ & $* * *$ & $* * *$ & $* * *$ \\
E\% & $*$ & $* * *$ & $* * *$ & $* * *$ & $* * *$ & $* * *$ \\
F\% & $* * *$ & $* * *$ & $* * *$ & $* * *$ & $*$ & $* * *$ \\
SCS & n.s. & n.s. & $* * *$ & $* * *$ & $* *$ & $* * *$ \\
\hline Irrtumswahrscheinlichkeit: n.s.: $\mathrm{p}>0,05 ; *: \mathrm{p} \leq 0,05 ;$ & $* *: \mathrm{p} \leq 0,01 ; * * * \mathrm{p} \leq 0,001$. & &
\end{tabular}

\section{Tabelle 3}

LS-Mittelwerte (LSM) und deren Standardfehler (SE) für die Milchmenge, den Fett- und Eiweißgehalt sowie den SCS der Bunten Deutschen Edelziegen in Abhängigkeit von der Region (LS-Means (LSM) and their standard errors (SE) of the milk yield, fat content, protein content and SCS of German Improved Fawn by region)

\begin{tabular}{cccccc}
\hline Region & $\begin{array}{c}\text { Anzahl Tiere/ } \\
\text { Beobachtungen }\end{array}$ & $\begin{array}{c}\text { Milch-kg } \\
\text { LSM } \pm \text { SE }\end{array}$ & $\begin{array}{c}\text { Fett-\% } \\
\text { LSM } \pm \text { SE }\end{array}$ & $\begin{array}{c}\text { Eiweiß-\% } \\
\text { LSM } \pm \text { SE }\end{array}$ & $\begin{array}{c}\text { SCS } \\
\text { LSM } \pm \text { SE }\end{array}$ \\
\hline $\begin{array}{c}\text { Nieder- } \\
\text { sachsen }\end{array}$ & $195 / 2857$ & $2,77 \pm 0,19^{\mathrm{a}}$ & $3,40 \pm 0,06^{\mathrm{a}}$ & $3,21 \pm 0,09^{\mathrm{a}}$ & $5,91 \pm 0,35^{\mathrm{a}}$ \\
$\begin{array}{c}\text { Sachsen } \\
\text { Baden- }\end{array}$ & $85 / 826$ & $2,98 \pm 0,24^{\mathrm{a}}$ & $3,54 \pm 0,08^{\mathrm{a}}$ & $3,26 \pm 0,13^{\mathrm{a}, \mathrm{b}}$ & $5,38 \pm 0,44^{\mathrm{a}}$ \\
Württemberg & $1568 / 24095$ & $3,00 \pm 0,05^{\mathrm{a}}$ & $3,09 \pm 0,01^{\mathrm{b}}$ & $3,45 \pm 0,03^{\mathrm{b}}$ & - \\
\hline
\end{tabular}

LSM mit unterschiedlichen Indizes unterscheiden sich signifikant voneinander $(\mathrm{p} \leq 0,05)$.

Tabelle 4

LS-Mittelwerte (LSM) und deren Standardfehler (SE) für die Milchmenge, den Fett- und Eiweißgehalt sowie den SCS der Bunten Deutschen Edelziegen in Abhängigkeit von der Anzahl lebend geborener Lämmer (LSMeans (LSM) and their standard errors (SE) of the milk yield, fat content, protein content and SCS of German Improved Fawn by number of kids born)

\begin{tabular}{cccccc}
\hline $\begin{array}{c}\text { Anzahl } \\
\text { Lämmer }\end{array}$ & $\begin{array}{c}\text { Anzahl Tiere/ } \\
\text { Beobachtungen }\end{array}$ & $\begin{array}{c}\text { Milch-kg } \\
\text { LSM } \pm \text { SE }\end{array}$ & $\begin{array}{c}\text { Fett-\% } \\
\text { LSM } \pm \text { SE }\end{array}$ & $\begin{array}{c}\text { Eiweiß-\% } \\
\text { LSM } \pm \text { SE }\end{array}$ & $\begin{array}{c}\text { SCS } \\
\text { LSM } \pm \text { SE }\end{array}$ \\
\hline 1 & $866 / 6307$ & $2,85 \pm 0,11^{\mathrm{a}}$ & $3,37 \pm 0,03^{\mathrm{a}}$ & $3,37 \pm 0,06^{\mathrm{a}}$ & $5,68 \pm 0,32^{\mathrm{a}}$ \\
2 & $2028 / 16095$ & $2,92 \pm 0,10^{\mathrm{b}}$ & $3,34 \pm 0,03^{\mathrm{b}}$ & $3,31 \pm 0,06^{\mathrm{b}}$ & $5,67 \pm 0,30^{\mathrm{a}}$ \\
$3-4$ & $553 / 4375$ & $2,99 \pm 0,11^{\mathrm{c}}$ & $3,33 \pm 0,03^{\mathrm{b}}$ & $3,24 \pm 0,06^{\mathrm{c}}$ & $5,59 \pm 0,31^{\mathrm{a}}$ \\
\hline
\end{tabular}

LSM mit unterschiedlichen Indizes unterscheiden sich signifikant voneinander $(\mathrm{p} \leq 0,05)$.

Tabelle 5

LS-Mittelwerte (LSM) und deren Standardfehler (SE) für die Milchmenge, den Fett- und Eiweißgehalt sowie den SCS der Bunten Deutschen Edelziegen in Abhängigkeit vom Ablammmonat (LS-Means (LSM) and their standard errors (SE) of the milk yield, fat content, protein content and SCS of German Improved Fawn by month of kidding)

\begin{tabular}{cccccc}
\hline $\begin{array}{c}\text { Ablamm- } \\
\text { monat }\end{array}$ & $\begin{array}{c}\text { Anzahl Tiere/ } \\
\text { Beobachtungen }\end{array}$ & $\begin{array}{c}\text { Milch-kg } \\
\text { LSM } \pm \text { SE }\end{array}$ & $\begin{array}{c}\text { Fett-\% } \\
\text { LSM } \pm \text { SE }\end{array}$ & $\begin{array}{c}\text { Eiweiß-\% } \\
\text { LSM } \pm \text { SE }\end{array}$ & $\begin{array}{c}\text { SCS } \\
\text { LSM } \pm \text { SE }\end{array}$ \\
\hline Januar & $1038 / 8706$ & $3,01 \pm 0,11^{\mathrm{a}}$ & $3,30 \pm 0,03^{\mathrm{a}}$ & $3,28 \pm 0,06^{\mathrm{a}}$ & $6,16 \pm 0,30^{\mathrm{a}}$ \\
Februar & $879 / 6900$ & $3,01 \pm 0,11^{\mathrm{a}}$ & $3,31 \pm 0,03^{\mathrm{a}}$ & $3,26 \pm 0,06^{\mathrm{a}}$ & $6,15 \pm 0,30^{\mathrm{a}}$ \\
März & $860 / 6408$ & $2,92 \pm 0,11^{\mathrm{b}}$ & $3,35 \pm 0,03^{\mathrm{b}}$ & $3,31 \pm 0,06^{\mathrm{b}}$ & $5,60 \pm 0,33^{\mathrm{b}}$ \\
April- & $797 / 5764$ & $2,73 \pm 0,11^{\mathrm{c}}$ & $3,42 \pm 0,03^{\mathrm{c}}$ & $3,38 \pm 0,06^{\mathrm{c}}$ & $4,67 \pm 0,39^{\mathrm{c}}$ \\
Dezember & & & & & \\
\hline
\end{tabular}

LSM mit unterschiedlichen Indizes unterscheiden sich signifikant voneinander $(\mathrm{p} \leq 0,05)$. 
Zwischen den einzelnen Regionen lagen signifikante Unterschiede hinsichtlich des Fett- und Eiweißgehaltes, nicht jedoch hinsichtlich der Milchmenge und des SCS vor (Tab. 3). Bunte Deutsche Edelziegen aus Sachsen zeichneten sich durch den höchsten Fettgehalt und Tiere aus Baden-Württemberg durch den höchsten Eiweißgehalt aus. Bunte Deutsche Edelziegen, die Zwillinge zur Welt brachten, produzierten signifikant mehr Milch als solche mit Einlingen (Tab. 4). Tiere mit drei oder vier Lämmern hatten wiederum eine signifikant höhere Milchleistung als solche mit Zwillingen. Mit steigender Anzahl Lämmer nahmen dagegen der Fett- und Eiweißgehalt der Milch signifikant ab. Der SCS erwies sich als unbeeinflusst von der Anzahl lebend geborener Lämmer. Die Milchmenge, der Fett- und Eiweißgehalt und der SCS variierten je nach Ablammmonat (Tab. 5). Allerdings waren die Unterschiede zwischen Tieren, die im Januar lammten, und Tieren, die ihre Lämmer im Februar zur Welt brachten, nicht signifikant. Zwischen den übrigen Ablammmonaten bestanden hingegen signifikante Unterschiede. So produzierten Bunte Deutsche Edelziegen, die später im Jahr lammten, signifikant weniger Milch als Tiere, die im Januar und Februar gelammt hatten. Jedoch stiegen der Fett- und Eiweißgehalt der Milch mit einer späteren Ablammung signifikant an. Der SCS von Ziegen, die im März oder später ablammten, war signifikant geringer als der von Ziegen, die im Januar und Februar lammten.

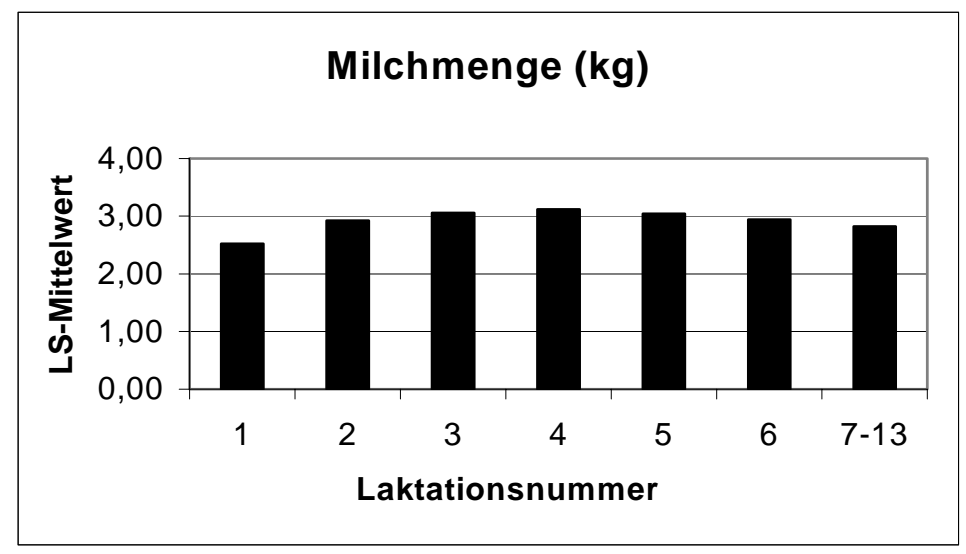

Abb. 5: LS-Mittelwerte der Milchmenge (kg) der Bunten Deutschen Edelziegen nach Laktationsnummern (LSMeans of the milk yield of German Improved Fawn by lactation number)

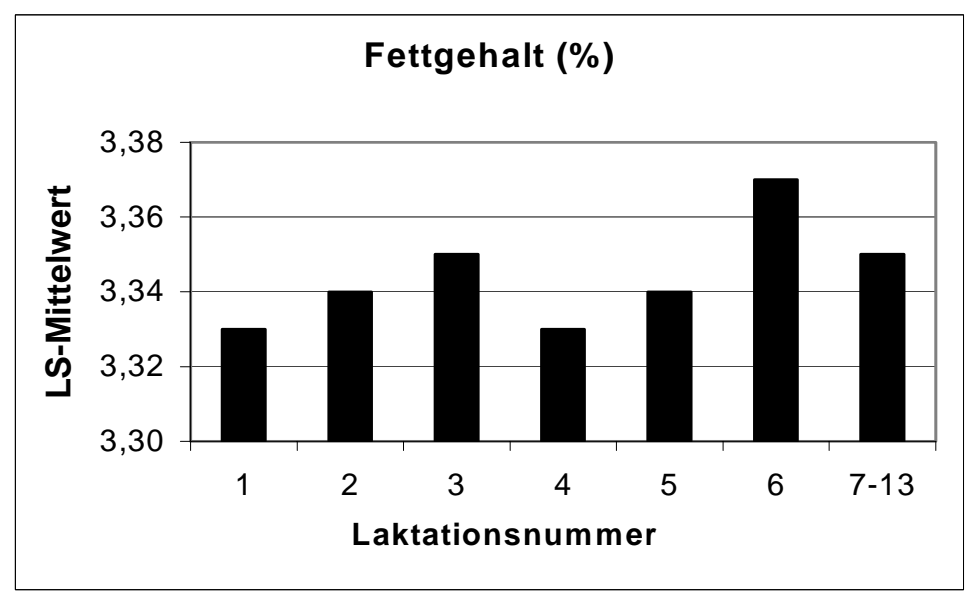

Abb. 6: LS-Mittelwerte des Fettgehaltes der Bunten Deutschen Edelziegen nach Laktationsnummern (LS-Means of the fat content of German Improved Fawn by lactation number) 


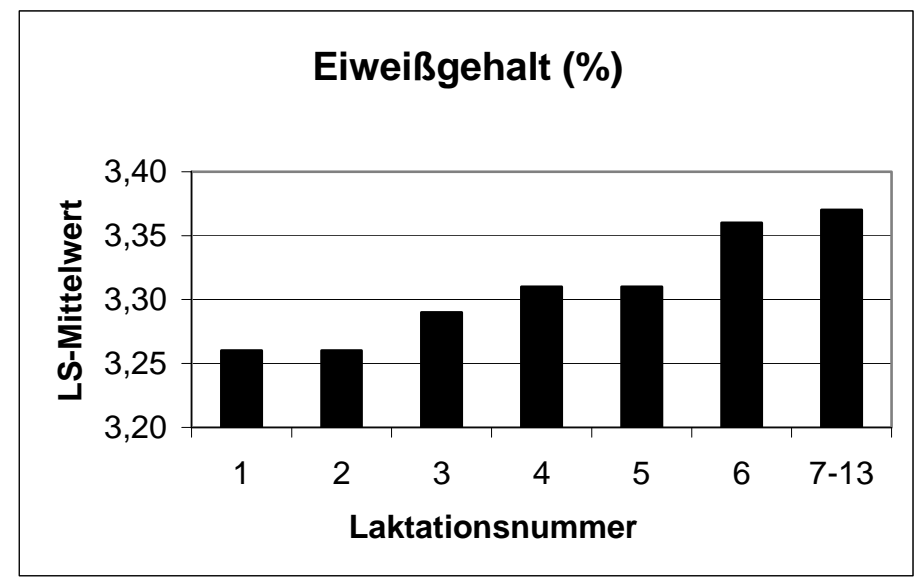

Abb. 7: LS-Mittelwerte des Eiweißgehaltes der Bunten Deutschen Edelziegen nach Laktationsnummern (LSMeans of the protein content of German Improved Fawn by lactation number)

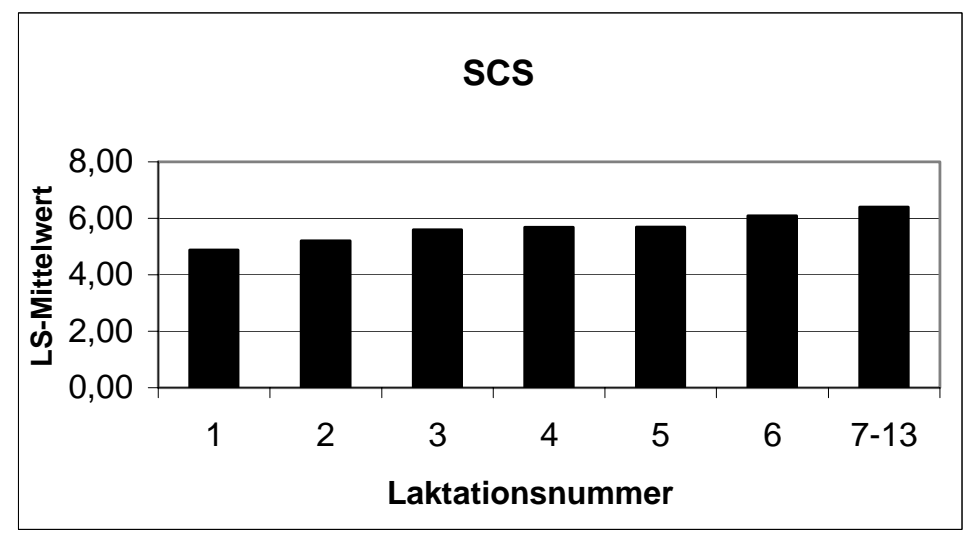

Abb. 8: LS-Schätzwerte des SCS der Bunten Deutschen Edelziegen nach Laktationsnummern (LS-Means of the somatic cell score (SCS) of German Improved Fawn for the effect of lactation number)

Von der ersten bis zur vierten Laktation kam es zu einem Anstieg der Milchmenge (Abb. 5). In den folgenden Laktationen nahm sie wieder ab. Dabei waren die Differenzen zwischen den Laktationsnummern, mit Ausnahme der Differenz zwischen der zweiten und der siebten sowie zwischen der dritten und der fünften Klasse, signifikant. Mit steigender Laktationsnummer nahm der Eiweißgehalt der Ziegenmilch zu (Abb. 6). Keine signifikanten Unterschiede bestanden jedoch zwischen der ersten und zweiten bzw. zwischen der vierten und fünften Laktation. Mit steigender Laktationsnummer nahm der Eiweißgehalt zu. Keine Unterschiede bestanden jedoch zwischen der ersten und zweiten bzw. zwischen der vierten und fünften Laktation. Signifikant waren der Anstieg von der fünften zur sechsten Laktation. Der Fettgehalt unterlag relativ großen Schwankungen. So stieg er von der ersten bis zur dritten Laktation an, fiel danach signifikant ab, nahm bis zur sechsten Laktation wieder zu und fiel zur letzten Laktationsnummernklasse hin nochmals ab. Signifikant waren die Differenzen zwischen der sechsten und allen anderen Laktationsnummernklassen mit Ausnahme der siebten sowie die Differenzen zwischen der dritten und vierten Klasse (Abb. 7). Der SCS der Bunten Deutschen Edelziegen stieg mit steigender Laktationsnummer kontinuierlich an. Signifikant waren dabei die Zunahmen von der ersten und zweiten zu allen späteren Laktationsnummern sowie zwischen der dritten, vierten und fünften und der siebten Laktationsnummernklasse. Auch die Differenz zwischen der dritten und der sechsten Klasse war signifikant (Abb. 8). 
4. Diskussion

Das Ziel der vorliegenden Arbeit war, die systematischen Effekte auf die Milchleistung von Bunten Deutschen Edelziegen zu beurteilen. Dies geschah für die Faktoren Region, Anzahl lebend geborener Lämmer, Ablammsaison, Ablammjahr, Laktationsnummer, Probemelkintervall, Tage in Milch, Betrieb und Tier.

Die Anzahl lebend geborener Lämmer erwies sich als signifikant für die Milch- und Fettmenge sowie für den Fett- und Eiweißgehalt. Keinen Einfluss hatte sie auf die Eiweißmenge und den SCS. Dies stimmt mit Beobachtungen von BROWNING et al. (1995) überein, nach denen Mehrlingsgeburten mit einer höheren Milchleistung einhergingen. Auch GALL (2001) beschrieb eine bessere Vorbereitung der folgenden Laktation bei Mehrlingsträchtigkeiten, die zu einer höheren Milchleistung führten. Bei den Bunten Deutschen Edelziegen wurde nicht nur bei Zwillingsgeburten im Vergleich zu Einlingsgeburten eine signifikant größere Milchmenge beobachtet, sondern es produzierten auch Ziegen, die Drillinge oder Vierlinge zu Welt brachten signifikant mehr Milch als solche mit Zwillingen. Gegenteilig verhielten sich dagegen die Gehalte der Milchinhaltstoffe, die im Vergleich von Einlings- zu Mehrlingsgeburten abnahmen. Ähnliche Tendenzen ergaben sich für die Analyse bei Weißen Deutschen Edelziegen (BÖMKES et al., 2004). Allerdings waren bei den Weißen Deutschen Edelziegen die Differenzen zwischen Tieren mit Einlings- und Mehrlingsgeburten ausgeprägter. Die Ursache für die Abnahme der Milchinhaltsstoffe bei Mehrlingen könnte in einem Verdünnungseffekt durch die ansteigende Milchmenge begründet sein. Eine Beeinflussung des SCS durch die Anzahl lebend geborener Lämmer wurde im Gegensatz zu den Weißen Deutschen Edelziegen nicht beobachtet (BÖMKES et al., 2004). Da die Mehrzahl der Lämmer, mit Ausnahme einiger Zuchtlämmer, nach der Geburt von der Mutter getrennt und mutterlos aufgezogen wurden, war mit einer Verschlechterung der Eutergesundheit nach Mehrlingsgeburten nicht zu rechnen.

Die Ablammsaison hatte einen signifikanten Einfluss auf alle Milchleistungsmerkmale der Bunten Deutschen Edelziegen. Auch ZOA-MBOE et al. (1997), BOICHARD et al. (1989), GROSSMANN und WIGGANS (1980) sowie CHERIX (1990) machten diese Beobachtungen für Ziegen unterschiedlicher Rassen. Bei den Weißen Deutschen Edelziegen zeigte sich ebenfalls ein signifikanter Einfluss der Ablammsaison auf alle Merkmale mit Ausnahme des Eiweißgehalts und SCS (BÖMKES et al., 2004). Wie bei den Weißen Deutschen Edelziegen fiel bei den Bunten Deutschen Edelziegen die Milchmenge umso deutlicher ab, je später der Zeitpunkt der Lammung war. Die Milchinhaltsstoffe stiegen hingegen mit späterer Lammzeit signifikant an. Ursache sind neben dem von LINZELL (1972) beschriebenen deutlichen Jahresrhythmus der Milchleistung, mit maximalen Leistungen in den Sommermonaten, auch Umwelteinflüsse, wie Fütterung (KIJORA et al., 2002), Temperatur und Weidegang (GALL, 2001). Das Ablammjahr hatte einen signifikanten Einfluss auf alle Milchleistungsmerkmale. Dies stimmt mit den Analysen bei Weißen Deutschen Edelziegen (BÖMKES et al., 2004) und den Ergebnissen von ZOA-MBOE et al. (1997) überein. Ursachen für die Veränderungen der Milchleistung zwischen den Ablammjahren können unter anderem Änderungen im Management, der Fütterung, der Melktechnik oder auch Klimaeinflüsse in feucht-heißen Gebieten sein. Signifikante Unterschiede bei allen Milchleistungsmerkmalen wurden weiterhin durch die Laktationsnummer verursacht. Die höchste Milchleistung erreichten Bunte Deutsche Edelziegen in der dritten und vierten Laktation. Auch GROSSMANN und WIGGANS (1980) und BOICHARD 
et al. (1989) beobachteten bei Ziegen verschiedener Rassen einen Anstieg der Milchmenge bis zur dritten oder vierten Laktation und einen darauffolgenden Abfall. Dass die Leistungen der Bunten Deutschen Edelziegen in der zweiten und den folgenden Laktationen höher waren als in der ersten Laktation, stimmte mit den Ergebnissen der Untersuchungen von ZOA-MBOE et al. (1997) und FINLEY et al. (1984) überein. Auch GALL (2001) und BÖMKES et al. (2004) beschrieben einen Anstieg der Milchmenge von der ersten bis zu dritten und späteren Laktation. Ebenso wie von GROSSMANN und WIGGANS (1980) und BOICHARD et al. (1989) berichtet, stieg auch bei den Bunten Deutschen Edelziegen die Fettmenge bis zur dritten bzw. vierten Laktation an und fiel danach wieder ab. Der Anstieg der Fettmenge ging mit einem Anstieg des Fettgehaltes in der Milch einher. Dagegen fiel bei den Weißen Deutschen Edelziegen der Fettgehalt von der ersten Laktation in den späteren Laktationen ab (BÖMKES et al., 2004). Der SCS nahm mit steigender Laktationsnummer in Übereinstimmung mit den Ergebnissen für die Weißen Deutschen Edelziegen (BÖMKES et al., 2004) signifikant zu. PERNTHANER et al. (1991) konnten dagegen keinen Anstieg der somatischen Zellzahl in Abhängigkeit von der Laktationsnummer beobachten, wohingegen DUNLIN et al. (1983) und FAHR et al. (1999, 2001) einen signifikanten Einfluss der Laktationsnummer auf den somatischen Zellgehalt nachwiesen.

Die Tage in Milch zeigten wie bei den Weißen Deutschen Edelziegen einen signifikanten Effekt auf alle untersuchten Milchleistungsmerkmale. LÖHLE und ZASTROW (1989) und GALL (2001) beschrieben ebenso einen Einfluss des Laktationsstadiums auf die Milchleistung von Milchziegen. Der Laktationskurvenverlauf zeigte weitgehend das gleiche Verhalten wie bei der Weißen Deutschen Edelziege (BÖMKES et al., 2004). Nach dem Lammen stieg die Milchmenge an, erreichte zwischen der zweiten und zehnten Woche ihr Maximum und fiel dann bis zum Laktationsende stetig ab. Durch den Rückgang der Milchmenge am Laktationsende stiegen die Gehalte der Milchinhaltstoffe in dieser Zeit stark an.

\section{Danksagung}

Der H. Wilhelm Schaumann Stiftung, Hamburg, sei für die Unterstützung dieser Arbeit sehr herzlich gedankt. Dem Ziegenzuchtverband Niedersachsen, dem Sächsischen Schaf- und Ziegenzuchtverband und dem Ziegenzuchtverband Baden-Württemberg danken wir sehr herzlich für die Datenbereitstellung.

\section{Literatur}

BÖMKES, D.; HAMANN, H.; DISTL, O.:

Schätzung genetischer Parameter für Testtagsergebnisse von Milchleistungsmerkmalen bei Bunten Deutschen Edelziegen. Arch. Tierz., Dummerstorf 47 (2004) 2, 193-202

BÖMKES, D.; HAMANN, H.; DISTL, O.:

Populationsgenetische Analyse von Milchleistungsmerkmalen bei Weißen Deutschen Edelziegen. 1. Mitteilung: Einfluss systematischer Umwelteffekte. Züchtungskunde 76 (2004), 127-138

BOICHARD, D.; BOULOG, N; RICORDEAU, G; PIACERE, A.; BARILLET; F.:

Genetic parameters for first lactation dairy traits in the Alpine and Saanen goat breeds. Génét. Sél. Evol. 21 (1989), 205-215

BROWNING, R.; LEITE-BROWNING, M.L.; SAHLU, T.:

Factors affecting standardized milk and fat yields in Alpine goats. Small Rumin. Res. 18 (1995), 173178 


\section{CHERIX, P.:}

Die Einflüsse einiger Umweltfaktoren auf die Milchleistung von Ziegen. Der Kleinviehzüchter 38 (1990), 67-74

DUNLIN, A.M.; PAAPE, M.J.; SCHULTZE, W.D.; WEINLAND, B.T.:

Effect of parity, stage of lactation, and intramammary infection on concentration of somatic cells and cytoplasmatic particles in goat milk. J. Dairy Sci. 66 (1983), 2426-2433

FAHR, R.-D.; SCHULZ, J.; FINN, G; LENGERKEN, G. v.; WALTHER, R.:

Zellgehalt und Differentialzellbild der Ziegenmilch - Variabilität und Einflussfaktoren. Tierärztl. Prax. 27 (1999), 99-106

FAHR, R.-D.; SÜß, R.; SCHULZ, J.; LENGERKEN, G. v.:

Vergleichende Untersuchungen zu Einflussfaktoren auf die somatische Zellzahl bei Schaf und Ziege. Arch. Tierz., Dummerstorf 44 (2001) Special Issue, 288-298

FINLEY, C.M.; THOMPSON, J.R.; BRADFORD, G.E.:

Age-Parity-Season Adjustment factors for milk and fat yields of dairy goats. J. Dairy Sci. 67 (1984), 1868-1872

GALL, C.:

Ziegenzucht. 2. Auflage, Ulmer Verlag, Stuttgart, 2001, 140-153

GROSSMANN, M.; WIGGANS, G.R.:

Dairy goat lactation records and potential for buck evaluation. J. Dairy Sci. 63 (1980), 1925-1937

KIJORA, C.; PETERS, K.-J.; REXROTH, H.; CHOWDHURY, S.:

Einfluss des Energie- und Proteinniveaus sowie der Proteinqualität auf die Milchleistung der Bunten Deutschen Edelziege. Arch. Tierz., Dummerstorf 45 (2002) 3, 255-268

LINZELL, J.L.:

Milk yield, energy loss in milk and mammary gland weight in different species. J. Dairy Sci. 34 (1972), $351-360$

LÖHLE, K.; ZASTROW, G.:

Untersuchungen über den Laktationskurvenverlauf bei Ziegen. Mh. Vet.-Med. 44 (1989), 757-759

PERTHANER A., PIROUTZ-PITTMANN, M.E.; SCHODER, G.; DEUTZ, A.; BAUMGARTNER, W.: Untersuchungen über den Verlauf des physiologischen Zellgehalts bei Ziegen während einer Laktation. Tierärztl. Umschau 48 (1993), 222-225

SAMBRAUS, H.H.:

Atlas der Nutztierrassen. 6. Auflage, Verlag Eugen Ulmer, Stuttgart (2001), 163-167

ZOA-MBOE, A.; MICHAUX, C.; DETILLEUX, J.C.; KEBERS, C.; FARNIR, F.P.; LEROY, P.L.:

Effects of parity, breed, herd-year, age, and month of kidding on the milk yield and composition of dairy goats in Belgium. J. Anim. Breed. Genet. 114 (1997), 201-213

Eingegangen: 08.10.2003

Akzeptiert: 17.05.2004

Anschrift der Verfasser

DOMINIKA BÖMKES, Dr. HENNING HAMANN, Prof. Dr. OTTMAR DISTL

Institut für Tierzucht und Vererbungsforschung der Tierärztliche Hochschule Hannover

Bünteweg 17p

D-30559 Hannover

E-Mail: ottmar.distl@tiho-hannover.de 